\title{
A Family of Modified Even Order Bernoulli-Type Multiquadric Quasi-Interpolants with Any Degree Polynomial Reproduction Property
}

\author{
Ruifeng Wu, Huilai Li, and Tieru Wu \\ School of Mathematics, Jilin University, Changchun 130012, China \\ Correspondence should be addressed to Tieru Wu; wutr@jlu.edu.cn
}

Received 5 November 2013; Accepted 14 January 2014; Published 10 March 2014

Academic Editor: Antonio J. M. Ferreira

Copyright (C) 2014 Ruifeng Wu et al. This is an open access article distributed under the Creative Commons Attribution License, which permits unrestricted use, distribution, and reproduction in any medium, provided the original work is properly cited.

By using the polynomial expansion in the even order Bernoulli polynomials and using the linear combinations of the shifts of the function $f(x)(x \in \mathbb{R})$ to approximate the derivatives of $f(x)$, we propose a family of modified even order Bernoulli-type multiquadric quasi-interpolants which do not require the derivatives of the function approximated at each node and can satisfy any degree polynomial reproduction property. Error estimate indicates that our operators could provide the desired precision by choosing a suitable shape-preserving parameter $c$ and a nonnegative integer $m$. Numerical comparisons show that this technique provides a higher degree of accuracy. Finally, applying our operators to the fitting of discrete solutions of initial value problems, we find that our method has smaller errors than the Runge-Kutta method of order 4 and Wang et al's quasi-interpolation scheme.

\section{Introduction}

Let $X=\left\{x_{0}, \ldots, x_{n}\right\}$ be a set of $n+1$ distinct points of $\mathbb{R}$ and let $f$ be a function defined on a domain $[a, b]$ containing $X$. The standard formula for interpolating the function $f$, where

$$
a=x_{0}<\cdots<x_{n}=b,
$$

has the following form:

$$
\mathscr{L}[f ; a, b](x)=\sum_{j=0}^{n} \lambda_{j} \mathscr{X}\left(x-x_{j}\right),
$$

s.t.

$$
\mathscr{L}[f ; a, b]\left(x_{j}\right)=f\left(x_{j}\right),
$$

for all $j=0, \ldots, n$, where $\mathscr{X}(\cdot)$ is an interpolation kernel. Many investigators use radial basis functions to solve the interpolation problems (2)-(3). In particular, the multiquadrics presented by Hardy [1],

$$
\phi_{j}(x)=\phi\left(x-x_{j}\right)=\sqrt{\left(x-x_{j}\right)^{2}+c^{2}}, \quad j=0, \ldots, n,
$$

are of especial interest because of their special convergence property, see $[2,3]$. Throughout this paper, let the notations $\phi_{j}(\cdot)$ and $c$ denote the multiquadrics and their shapepreserving parameter as in (4), respectively. A review by Franke [4] indicated that the multiquadric interpolation is one of the best schemes among some 29 interpolation methods in terms of accuracy, efficiency, and easy implementation. Although the multiquadric interpolation is always solvable when the scattered points $\left\{x_{j}\right\}_{j=0}^{n}$ are distinct [5], the resulting matrix in (2)-(3) quickly becomes ill-conditioned as the number of the scattered points increases. In this paper, we will use the quasi-interpolation technique to overcome the illconditioning problem.

A weaker form of (3), well-known as quasi-interpolation, holds for all polynomials of degree no more than $m$, where $m$ is nonnegative integer; that is,

$$
\mathscr{L}[p ; a, b]\left(x_{j}\right)=p\left(x_{j}\right), \quad j=0, \ldots, n, p \in \mathbb{P}^{m},
$$

where $\mathbb{P}^{m}=\{p: \operatorname{deg}(p) \leqslant m\}$. Beatson and Powell [6] first proposed a univariate quasi-interpolation operator $\mathscr{L}_{B}$ which reproduces constants, where $\mathscr{X}(x)$ in $(2)$ is a linear combination of the multiquadrics, see (46)-(47). 
Afterwards, Wu and Schaback [7] proposed another quasiinterpolation operator $\mathscr{L}_{D}$ which possesses shape-preserving and linear reproducing properties. They showed that the error of the operator $\mathscr{L}_{D}$ is $\mathcal{O}\left(h^{2}|\ln h|\right)$, when the shape parameter $c=\mathcal{O}(h)$, where $h=\max _{1 \leqslant j \leqslant n}\left\{\left|x_{j}-x_{j-1}\right|\right\}$. Using the operator $\mathscr{L}_{D}$, Ling [8] constructed a multilevel quasi-interpolation operator and proved that its convergence order is $\mathscr{O}\left(h^{2.5}|\ln h|\right)$ when $c=\mathscr{O}(h)$. Using the shifts of the cubic multiquadrics, Feng and Li [9] constructed a shapepreserving quasi-interpolation operator. They showed that the operator reproduces all polynomials of degree 2 or less and proved that the convergence rate is $\mathcal{O}\left(h^{3}\right)$ as $c=\mathcal{O}\left(h^{1.5}\right)$. Combining the operator $\mathscr{L}_{B}$ with Hermite interpolation polynomials, Wang et al. [10] proposed a kind of improved quasi-interpolation operators $\mathscr{L}_{H_{2 m-1}}$ which reproduce all polynomials of degree $\leqslant 2 m-1$. They proved that it converges with a rate of $\mathcal{O}\left(h^{2 m}\right)$ at most. However, the operators $\mathscr{L}_{H_{2 m-1}}$ require values of the derivatives at endpoints, which are not convenient for practical purposes. Further, many authors offered some examples using multiquadric quasiinterpolation operator to solve differential equations, see [1115] for details.

Based on CAIRA-DELL'ACCIO's idea [16], we first define a family of even order Bernoulli-type multiquadric quasiinterpolants $\widetilde{\mathscr{L}}_{v_{m}}$ by combining the multiquadric quasiinterpolation operator $\mathscr{L}_{B}$ in [6] with the polynomial expansion in even order Bernoulli polynomials $v_{m}(x)$ in [17]. For practical purposes, applying the divided difference formula in [18] to the operators $\widetilde{\mathscr{L}}_{v_{m}}$, we construct a family of modified even order Bernoulli-type multiquadric quasi-interpolants $\mathscr{L}_{v_{m}}$ which do not require values of the derivatives at nodes. We prove that the operators $\mathscr{L}_{v_{m}}$ reproduce all polynomials of degree $\leqslant 2 m$ and have the convergence rate of $\mathcal{O}\left(h^{2 m+1}\right)$ under a suitable assumption on the shape parameter $c$. Therefore, our operators $\mathscr{L}_{v_{m}}$ can provide the desired smoothness and precision in the practical applications.

The organization of the remainder of this paper is as follows. In Section 2, we briefly recall the definition of Bernoulli polynomials and even order Bernoulli polynomials giving some useful properties. We also obtain three useful theorems for the error in the even order Bernoulli polynomials expansion. In Section 3, we apply previous results to derive a family of modified even order Bernoulli-type multiquadric quasi-interpolants and get their convergence rate. In Section 4, numerical examples are shown to compare the approximation capacity of our new operators with that of CAIRA-DELL'ACCIO's interpolants and Wang et al.'s quasiinterpolants. In Section 5, we apply our operators to the fitting of discrete solutions of initial value problems for ordinary differential equations. In Section 6, we give the conclusions.

\section{Some Remarks on the Polynomial Expansion}

2.1. The Generalized Taylor Polynomial. The generalized Taylor polynomial is an expansion in Bernoulli polynomials
$B_{n}(x)$, that is, the polynomials of the sequence defined recursively by means of the following relations, see [19]:

$$
\begin{gathered}
B_{0}(x)=1, \\
B_{n}^{\prime}(x)=n B_{n-1}(x), \quad n \geqslant 1, \\
\int_{0}^{1} B_{n}(x) d x=0, \quad n \geqslant 1 .
\end{gathered}
$$

Let function $f(x)$ be in the class $C^{m}[a, b](a<b)$; then

$$
f(x)=P_{m}[f ; a, b](x)+R_{m}[f ; a, b](x), \quad x \in[a, b],
$$

where the polynomial approximation term is considered as follows:

$$
\begin{aligned}
P_{m}[f ; a, b](x) & \\
=f(a)+\sum_{k=1}^{m} & \frac{B_{k}((x-a) / h)-B_{k}(0)}{k !} \\
& \times h^{k-1}\left(f^{(k-1)}(b)-f^{(k-1)}(a)\right)
\end{aligned}
$$

and the remainder term is defined by

$$
\begin{aligned}
& R_{m}[f ; a, b](x) \\
& =\frac{h^{m-1}}{m !} \int_{a}^{b} f^{(m)}(t) \\
& \quad \times\left(B_{m}\left(\frac{b-t}{h}\right)-B_{m}\left(\frac{x-t-[x-t]}{h}\right)\right) d t
\end{aligned}
$$

where $[\cdot]$ denotes the integer part of the argument and $h=$ $b-a$. The polynomial approximant $P_{m}[f ; a, b](x)$ has the following result:

$$
\lim _{h \rightarrow 0} P_{m}[f ; a, b](x)=T_{m}[f ; a](x),
$$

where $T_{m}[f ; a](x)$ is the $r$ th Taylor polynomial of $f$ with initial point in $a$. According to (10), we denote by $P_{m}[f ; a, b](x)$ the generalized Taylor polynomial.

2.2. The Polynomial Expansion in Even Order Bernoulli Polynomials. Let us consider the polynomial sequence defined recursively by the following relations, see [17]:

$$
\begin{gathered}
v_{0}(x)=1 \\
v_{n}^{\prime}(x)=\int_{0}^{x} v_{n-1}(t) d t, \quad n=1,2, \ldots \\
\int_{0}^{1} v_{n}(x) d x=0, \quad n=1,2, \ldots
\end{gathered}
$$

By (11), the polynomial sequence $\left\{v_{n}(x)\right\}$ is related to the following Bernoulli polynomials of even degree, see [17]:

$$
v_{n}(x)=\frac{2^{2 n}}{(2 n) !} B_{2 n}\left(\frac{1+x}{2}\right), \quad n=1,2, \ldots
$$


We denote by $v_{n}(x)$ the even order Bernoulli polynomials. For any function $f$ in the class $C^{2 m}[a, b](a<b)$, this expansion is realized by the following:

$$
f(x)=P_{a, m}[f ; a, b](x)+R_{a, m}[f ; a, b](x), \quad x \in[a, b],
$$

where the polynomial expansion $P_{a, m}[f ; a, b](x)$ in even order Bernoulli polynomials is defined by

$$
\begin{aligned}
P_{a, m}[f ; a, b](x) & \\
=f(a)+\sum_{j=1}^{m}[ & h^{2 j-1} f^{(2 j-1)}(b)\left(v_{j}\left(\frac{x-a}{h}\right)-v_{j}(0)\right) \\
& \left.\quad-h^{2 j-1} f^{(2 j-1)}(a)\left(v_{j}\left(\frac{b-x}{h}\right)-v_{j}(1)\right)\right]
\end{aligned}
$$

and the remainder $R_{a, m}[f ; a, b](x)$ in its Peano's representation is given by

$$
R_{a, m}[f ; a, b](x)=h^{2 m-1} \int_{a}^{b} f^{(2 m)}(t) K_{a, m}\left(\frac{x-a}{h}, \frac{t-a}{h}\right) d t,
$$

where

$$
\begin{aligned}
& K_{a, m}(x, t) \\
&=\left\{\begin{array}{cl}
-\sum_{j=1}^{m}\left(v_{j}(x)-v_{j}(0)\right) \frac{(1-t)^{2 m-2 j}}{(2 m-2 j) !}, & x \leqslant t, \\
\frac{t^{2 m-1}}{(2 m-1) !}+\sum_{j=1}^{m}\left(v_{j}(1-x)-v_{j}(1)\right) & x \geqslant t . \\
\times \frac{t^{2 m-2 j}}{(2 m-2 j) !}, &
\end{array}\right.
\end{aligned}
$$

In order to get bounds for remainder (15) even in points outside the interval $[a, b]$, we consider the operator

$$
f \longrightarrow P_{a, m}[f ; a, b],
$$

where $f \in C^{m}[c, d]$ with $c<a$ and $b<d$. By applying Peano's kernel theorem [20], we give an integral expression for the remainder (15) as follows.

Theorem 1. If $f \in C^{2 m}[c, d]$ and $x \in[c, d]$, then for the remainder

$$
R_{a, m}[f ; a, b](x)=f(x)-P_{a, m}[f ; a, b](x)
$$

we have the following integral representations:

$$
\begin{aligned}
R_{a, m}[f ; a, b](x) & \\
= & \begin{array}{ll}
\frac{1}{(2 m-1) !} \int_{x}^{b} f^{(2 m)}(t) K_{a, b}(x, t) d t, & c \leqslant x \leqslant a, \\
\frac{1}{(2 m-1) !} \int_{a}^{b} f^{(2 m)}(t) K_{a, b}(x, t) d t, & a \leqslant x \leqslant b, \\
\frac{1}{(2 m-1) !} \int_{a}^{x} f^{(2 m)}(t) K_{a, b}(x, t) d t, & b \leqslant x \leqslant d,
\end{array}
\end{aligned}
$$

where

$$
\begin{aligned}
& K_{a, b}(x, t) \\
& =(x-t)_{+}^{2 m-1}-(a-t)_{+}^{2 m-1} \\
& -\sum_{j=1}^{m}\left[h^{2 j-1} \frac{(2 m-1) !}{(2 m-2 j) !}(b-t)_{+}^{2 m-2 j}\left(v_{j}\left(\frac{x-a}{h}-v_{j}(0)\right)\right)\right. \\
& \quad-h^{2 j-1} \frac{(2 m-1) !}{(2 m-2 j) !}(a-t)_{+}^{2 m-2 j} \\
& \left.\quad \times\left(v_{j}\left(\frac{b-x}{h}\right)-v_{j}(1)\right)\right]
\end{aligned}
$$

and $(\cdot)_{+}^{k}$ denotes the positive part of the $k$ th power of the argument; that is,

$$
(s)_{+}^{k}=\max \left\{s^{k}, 0\right\} .
$$

Proof. On one hand, there are evaluations of derivatives of $f$ up to the order $2 m-1$ on points $a$ and $b$ of $[c, d]$ in the approximation term (14); on the other hand, the exactness of (14) on the set $\mathbb{P}^{2 m}$ denotes the exactness of the operator $P_{a, m}[f ; a, b]$ on the subset $\mathbb{P}^{2 m-1}$. Applying Peano's kernel theorem, we then obtain

$$
R_{a, m}[f ; a, b](x)=\frac{1}{(2 m-1) !} \int_{c}^{d} f^{(2 m)}(t) K_{a, b}(x, t) d t,
$$

where (20) is given by applying the linear functional $f \rightarrow$ $R_{a, m}[f ; a, b](x)$ to a function $(x-t)_{+}^{2 m-1}$ in $x$. Let $x \in[c, a]$; then

$$
\begin{aligned}
R_{a, m}[f ; a, b](x)= & \frac{1}{(2 m-1) !} \int_{c}^{x} f^{(2 m)}(t) K_{a, b}(x, t) d t \\
& +\frac{1}{(2 m-1) !} \int_{x}^{a} f^{(2 m)}(t) K_{a, b}(x, t) d t \\
& +\frac{1}{(2 m-1) !} \int_{a}^{b} f^{(2 m)}(t) K_{a, b}(x, t) d t \\
& +\frac{1}{(2 m-1) !} \int_{b}^{d} f^{(2 m)}(t) K_{a, b}(x, t) d t .
\end{aligned}
$$

Let $t \in[c, x]$; then

$$
\begin{aligned}
& K_{a, b}(x, t) \\
& =(x-t)^{2 m-1}-(a-t)^{2 m-1} \\
& -\left[\sum_{j=1}^{m} \frac{(2 m-1) !}{(2 m-2 j) !}(b-t)^{2 m-2 j} h^{2 j-1}\left(v_{j}\left(\frac{x-a}{h}\right)-v_{j}(0)\right)\right. \\
& \left.\quad+\frac{(2 m-1) !}{(2 m-2 j) !}(a-t)^{2 m-2 j}\left(v_{j}\left(\frac{b-x}{h}-v_{j}(1)\right)\right)\right]=0,
\end{aligned}
$$


where $(x-t)^{2 m-1}$ is considered a polynomial in $x$ of degree $2 m-1$. By the expression of $(\cdot)_{+}^{k},(20)$ is equal to zero in the interval $b<t<d$. Thus, we prove the first case of (19). The remaining cases of (19) can be got in an analogous manner.

By Theorem 1, we can get the following result.

Theorem 2. If $f \in C^{2 m}[c, d]$ and $x \in[c, d]$, then for the remainder (18) we get

$\left|R_{a, m}[f ; a, b](x)\right| \leqslant \begin{cases}C(m)\left\|f^{(2 m)}\right\|_{\infty}(b-x)^{2 m}, & c<x<a, \\ C(m)\left\|f^{(2 m)}\right\|_{\infty}(b-a)^{2 m}, & a<x<b, \\ C(m)\left\|f^{(2 m)}\right\|_{\infty}(x-a)^{2 m}, & b<x<d,\end{cases}$

where $\|\cdot\|_{\infty}$ denotes the sup-norm on $[c, d]$ and

$$
\begin{aligned}
& C(m) \\
& =\frac{1}{(2 m) !}\left[2^{2 m-1}+\sum_{j=1}^{m} \sum_{k=1}^{2 j-1} \frac{2^{k+1}-2}{2 m-2 j+1}\right. \\
& \left.\times\left(\begin{array}{c}
2 m \\
2 j
\end{array}\right)\left(\begin{array}{c}
2 j \\
k
\end{array}\right)\left|B_{k}(1)\right|\right] .
\end{aligned}
$$

Proof. Let $c<x<a$; then we find from (19) that

$$
\begin{aligned}
R_{a, m}[f ; a, b](x)= & \frac{1}{(2 m-1) !} \int_{x}^{\alpha} f^{(2 m)}(t) K_{a, b}(x, t) d t \\
& +\frac{1}{(2 m-1) !} \int_{a}^{b} f^{(2 m)}(t) K_{a, b}(x, t) d t .
\end{aligned}
$$

Let $x<t<a$; then

$$
\begin{aligned}
& K_{a, b}(x, t) \\
& =-(a-t)^{2 m-1}-\sum_{j=1}^{m}\left[\frac{(2 m-1) !}{(2 m-2 j) !}(b-t)^{2 m-2 j} h^{2 j-1}\right. \\
& \times\left(v_{j}\left(\frac{x-a}{h}\right)-v_{j}(0)\right) \\
& -\frac{(2 m-1) !}{(2 m-2 j) !}(a-t)^{2 m-2 j} h^{2 j-1} \\
& \left.\times\left(v_{j}\left(\frac{b-x}{h}\right)-v_{j}(1)\right)\right]
\end{aligned}
$$

so that

$$
\begin{aligned}
& \int_{x}^{a} K_{a, b}(x, t) f^{(2 m)}(t) d t \\
& =-\int_{x}^{a}(a-t)^{2 m-1} f^{(2 m)}(t) d t \\
& \quad-\sum_{j=1}^{m} \frac{(2 m-1) !}{(2 m-2 j) !} h^{2 j-1} \\
& \quad \times \int_{x}^{a}\left[(b-t)^{2 m-2 j}\left(v_{j}\left(\frac{x-a}{h}\right)-v_{j}(0)\right)\right. \\
& \left.\quad \quad-(a-t)^{2 m-2 j}\left(v_{j}\left(\frac{b-x}{h}\right)-v_{j}(1)\right)\right] f^{(2 m)}(t) d t .
\end{aligned}
$$

In [21], we have the following known identity:

$$
B_{n}(z+w)=\sum_{i=0}^{n}\left(\begin{array}{c}
n \\
i
\end{array}\right) B_{i}(z) w^{n-i}, \quad n=0,1, \ldots
$$

By the identities [17], using relations (30), we get

$$
\begin{aligned}
v_{n}(x) & =\frac{2^{2 n}}{(2 n) !} B_{2 n}\left(\frac{1+x}{2}\right)=\frac{2^{2 n}}{(2 n) !} B_{2 n}\left(\frac{1}{2}+\frac{x}{2}\right) \\
& =\frac{2^{2 n}}{(2 n) !} \sum_{i=0}^{2 n}\left(\begin{array}{c}
2 n \\
i
\end{array}\right) B_{i}\left(\frac{1}{2}\right)\left(\frac{x}{2}\right)^{2 n-i}, \quad n=0,1, \ldots
\end{aligned}
$$

In [17], we have

$$
\begin{gathered}
B_{0}(1)=B_{0}\left(\frac{1}{2}\right)=1, \\
B_{1}(0)=-\frac{1}{2}, \quad B_{1}(1)=\frac{1}{2}, \\
B_{n}\left(\frac{1}{2}\right)=-\left(1-2^{1-n}\right) B_{n}(0), \quad n=1,2, \ldots, \\
B_{n}(0)=B_{n}(1), \quad n=2, \ldots, \\
B_{2 n+1}(0)=0, \quad n=1,2, \ldots
\end{gathered}
$$

Therefore, by applying (31), we obtain the following form from (29):

$$
\begin{aligned}
& v_{j}\left(\frac{x-a}{h}\right)-v_{j}(0) \\
& =\frac{2^{2 j}}{(2 j) !} \sum_{k=0}^{2 j-1}\left(\begin{array}{c}
2 j \\
k
\end{array}\right) B_{k}\left(\frac{1}{2}\right)\left(\frac{x-a}{2 h}\right)^{2 j-k}, \quad j=1,2, \ldots, \\
& v_{j}\left(\frac{b-x}{h}\right)-v_{j}(1) \\
& =\frac{2^{2 j}}{(2 j) !} \sum_{k=0}^{2 j-1}\left(\begin{array}{c}
2 j \\
k
\end{array}\right) B_{k}(1)\left(\frac{a-x}{2 h}\right)^{2 j-k}, \quad j=1,2, \ldots
\end{aligned}
$$


Further, by applying the third case of (32) and the identities (33), we have

$$
\begin{aligned}
& \int_{x}^{a} K_{a, b}(x, t) f^{(2 m)}(t) d t \\
& =-\int_{x}^{a}(a-t)^{2 m-1} f^{(2 m)}(t) d t \\
& -\sum_{j=1}^{m} \frac{(2 m-1) !}{(2 m-2 j) !} \frac{2^{2 j}}{(2 j) !}\left(\frac{x-a}{2 h}\right)^{2 j} h^{2 j-1} \\
& \quad \times \int_{x}^{a}\left((b-t)^{2 m-2 j}-(a-t)^{2 m-2 j}\right) f^{(2 m)}(t) d t \\
& -\sum_{j=1}^{m} \sum_{k=1}^{2 j-1} \frac{(2 m-1) !}{(2 m-2 j) !} \frac{2^{2 j}}{(2 j) !} B_{k}\left(\frac{1}{2}\right)\left(\frac{x-a}{2 h}\right)^{2 j-k} h^{2 j-1} \\
& \quad \times \int_{x}^{a}(b-t)^{2 m-2 j} f^{(2 m)}(t) d t \\
& +\sum_{j=1}^{m} \sum_{k=1}^{2 j-1} \frac{(2 m-1) !}{(2 m-2 j) !} \frac{2^{2 j}}{(2 j) !} B_{k}(1)\left(\frac{a-x}{2 h}\right)^{2 j-k} h^{2 j-1} \\
& \quad \times \int_{x}^{a}(a-t)^{2 m-2 j} f^{(2 m)}(t) d t .
\end{aligned}
$$

Note that the integrands are of type $g(t) f^{(2 m)}(t)$ with a $g(t)$ that does not change sign in $[x, a]$. By applying the first mean value theorem for integrals to (34), we find for some $\xi, \xi_{j}, \eta_{j}$, $\theta_{j} \in[c, d], j=1, \ldots, m$ that

$$
\begin{aligned}
& \int_{x}^{a} K_{a, b}(x, t) f^{(2 m)}(t) d t \\
& =-f^{(2 m)}(\xi) \int_{x}^{a}(a-t)^{2 m-1} d t \\
& -\sum_{j=1}^{m} \frac{(2 m-1) !}{(2 m-2 j) !} \frac{2^{2 j}(2 j) !}{\left(\frac{x-a}{2 h}\right)^{2 j} h^{2 j-1} f^{(2 m)}\left(\xi_{j}\right)} \\
& \quad \times \int_{x}^{a}\left((b-t)^{2 m-2 j}-(a-t)^{2 m-2 j}\right) d t \\
& -\sum_{j=1}^{m} \sum_{k=1}^{2 j-1} \frac{(2 m-1) !}{(2 m-2 j) !} \frac{2^{2 j}}{(2 j) !} B_{k}\left(\frac{1}{2}\right)\left(\frac{x-a}{2 h}\right)^{2 j-k} \\
& \quad \times h^{2 j-1} f^{(2 m)}\left(\eta_{j}\right) \int_{x}^{a}(b-t)^{2 m-2 j} d t \\
& +\sum_{j=1}^{m} \sum_{k=1}^{2 j-1} \frac{(2 m-1) !}{(2 m-2 j) !} \frac{2^{2 j}}{(2 j) !} B_{k}(1)\left(\frac{a-x}{2 h}\right)^{2 j-k} \\
& \times h^{2 j-1} f^{(2 m)}\left(\theta_{j}\right) \int_{x}^{a}(a-t)^{2 m-2 j} d t .
\end{aligned}
$$

After some calculations in (35), we obtain

$$
\begin{aligned}
& \int_{x}^{a} K_{a, b}(x, t) f^{(2 m)}(t) d t \\
& =-f^{(2 m)}(\xi) \frac{(a-x)^{2 m}}{2 m} \\
& -h^{2 m} \sum_{j=1}^{m} \frac{(2 m-1) !}{(2 m-2 j+1) !(2 j) !}\left(\frac{x-a}{h}\right)^{2 j} f^{(2 m)}\left(\xi_{j}\right) \\
& \times\left[-1+\sum_{r=0}^{2 m-2 j}\left(\frac{b-x}{h}\right)^{2 m-2 j-r}\left(\frac{a-x}{h}\right)^{r}\right] \\
& -h^{2 m} \sum_{j=1}^{m} \sum_{k=1}^{2 j-1} \frac{(2 m-1) ! 2^{k}}{(2 m-2 j) !(2 j !)}\left(\begin{array}{c}
2 j \\
k
\end{array}\right) B_{k}\left(\frac{1}{2}\right)\left(\frac{x-a}{h}\right)^{2 j-k} \\
& \quad \times f^{(2 m)}\left(\eta_{j}\right)\left[1-\left(\frac{b-x}{h}\right)^{2 m-2 j+1}\right] \\
& +h^{2 m} \sum_{j=1}^{m} \sum_{k=1}^{2 j-1} \frac{(2 m-1) ! 2^{k}}{(2 m-2 j+1) !(2 j) !}\left(\begin{array}{c}
2 j \\
k
\end{array}\right) B_{k}(1) \\
& \times\left(\frac{a-x}{h}\right)^{2 j-k} f^{(2 m)}\left(\theta_{j}\right)\left(\frac{a-x}{h}\right)^{2 m-2 j+1} .
\end{aligned}
$$

Let $a<t<b$; then we have

$$
\begin{aligned}
& K_{a, b}(x, t)=-\sum_{j=1}^{m} \frac{(2 m-1) !}{(2 m-2 j) !}(b-t)^{2 m-2 j} h^{2 j-1} \\
& \quad \times\left(v_{j}\left(\frac{x-a}{h}\right)-v_{j}(0)\right), \\
& \int_{a}^{b} K_{a, b}(x, t) f^{(2 m)}(t) d t \\
& =-\sum_{j=1}^{m} \sum_{k=0}^{2 j-1} \frac{(2 m-1) !}{(2 m-2 j) !} h^{2 j-1}\left(\begin{array}{c}
2 j \\
k
\end{array}\right) B_{k}\left(\frac{1}{2}\right) \\
& \quad \times\left(\frac{x-a}{h}\right)^{2 j-k} \int_{x}^{a}(b-t)^{2 m-2 j} f^{(2 m)}(t) d t .
\end{aligned}
$$

By the first mean value theorem for integrals, we can get after some calculations

$$
\begin{aligned}
\int_{a}^{b} & K_{a, b}(x, t) f^{(2 m)}(t) d t \\
=-h^{2 m} \sum_{j=1}^{m} \sum_{k=0}^{2 j-1} & \frac{(2 m-1) ! 2^{k}}{(2 m-2 j+1) !(2 j) !}\left(\begin{array}{c}
2 j \\
k
\end{array}\right) B_{k}\left(\frac{1}{2}\right) \\
& \times\left(\frac{x-a}{h}\right)^{2 j-k} f^{(2 m)}\left(\lambda_{j}\right),
\end{aligned}
$$


where $\lambda_{j} \in[c, d], j=1, \ldots, m$. By applying relations (36) and (38) to (27), we have

$$
\begin{aligned}
& R_{a, m}[f ; a, b](x) \\
& =-f^{(2 m)}(\xi) \frac{(a-x)^{2 m}}{(2 m) !} \\
& -\frac{h^{2 m}}{(2 m) !} \sum_{j=1}^{m} \frac{(2 m) !}{(2 m-2 j+1) !(2 j) !}\left(\frac{x-a}{h}\right)^{2 j} f^{(2 m)}\left(\xi_{j}\right) \\
& \times\left[-1+\sum_{r=0}^{2 m-2 j}\left(\frac{b-x}{h}\right)^{2 m-2 j+r}\left(\frac{a-x}{h}\right)^{r}\right] \\
& -\frac{h^{2 m}}{(2 m) !} \sum_{j=1}^{m} \sum_{k=1}^{2 j-1} \frac{(2 m) ! 2^{k}}{(2 m-2 j+1) !(2 j) !}\left(\begin{array}{c}
2 j \\
k
\end{array}\right) B_{k}\left(\frac{1}{2}\right) \\
& \times\left(\frac{x-a}{h}\right)^{2 j-k} f^{(2 m)}\left(\eta_{j}\right) \\
& \times\left[1-\left(\frac{b-x}{h}\right)^{2 m-2 j+1}\right] \\
& +\frac{h^{2 m}}{(2 m) !} \sum_{j=1}^{m} \sum_{k=1}^{2 j-1} \frac{(2 m) ! 2^{k}}{(2 m-2 j+1) !(2 j) !}\left(\begin{array}{c}
2 j \\
k
\end{array}\right) B_{k}(1) \\
& \times\left(\frac{a-x}{h}\right)^{2 m-k+1} f^{(2 m)}\left(\theta_{j}\right)-\frac{h^{2 m}}{(2 m) !} \\
& \times \sum_{j=1}^{m} \sum_{k=0}^{2 j-1} \frac{(2 m) ! 2^{k}}{(2 m-2 j+1) !(2 j) !}\left(\begin{array}{c}
2 j \\
k
\end{array}\right) B_{k}\left(\frac{1}{2}\right) \\
& \times\left(\frac{x-a}{h}\right)^{2 j-k} f^{(2 m)}\left(\lambda_{j}\right) .
\end{aligned}
$$

By identities (32), we obtain

$$
\begin{gathered}
\left|B_{0}\left(\frac{1}{2}\right)\right|=\left|B_{0}(1)\right|=1, \\
\left|B_{n}\left(\frac{1}{2}\right)\right|=\frac{2^{n}-2}{2^{n}}\left|B_{n}(1)\right|, \quad n=1,2, \ldots
\end{gathered}
$$

By using (40) in (39), we have after some simplifications that

$$
\begin{aligned}
& \left|R_{a, m}[f ; a, b](x)\right| \\
& \leqslant \frac{\left\|f^{(2 m)}\right\|_{\infty}}{(2 m) !}\left((a-x)^{2 m}+h^{2 m} \sum_{j=1}^{m}\left(\begin{array}{c}
2 m \\
2 j
\end{array}\right)\left(\frac{b-x}{h}\right)^{2 m}\right.
\end{aligned}
$$

$$
\begin{gathered}
+h^{2 m} \sum_{j=1}^{m} \sum_{k=1}^{2 j-1} \frac{2^{k+1}-2}{2 m-2 j+1}\left(\begin{array}{c}
2 m \\
2 j
\end{array}\right)\left(\begin{array}{c}
2 j \\
k
\end{array}\right)\left|B_{k}(1)\right| \\
\left.\times\left(\frac{b-x}{h}\right)^{2 m+1-k}\right) \\
\leqslant h^{2 m} \frac{\left\|f^{(2 m)}\right\|_{\infty}}{(2 m) !}\left(1+\sum_{j=1}^{m}\left(\begin{array}{c}
2 m \\
2 j
\end{array}\right)+\sum_{j=1}^{m} \sum_{k=1}^{2 j-1} \frac{2^{k+1}-2}{2 m-2 j+1}\left(\begin{array}{c}
2 m \\
2 j
\end{array}\right)\right. \\
\left.\times\left(\begin{array}{c}
2 j \\
k
\end{array}\right)\left|B_{k}(1)\right|\right)\left(\frac{b-x}{h}\right)^{2 m} .
\end{gathered}
$$

Because

$$
1+\sum_{j=1}^{m}\left(\begin{array}{c}
2 m \\
2 j
\end{array}\right)=2^{2 m-1}
$$

we obtain the first case of expression (25). Similarly, we can prove the remaining cases.

Since the polynomials $P_{a, m}[f ; a, b](x)$ of degree are not greater than $2 m$, we can obtain the desired bounds in an analogous manner.

Theorem 3. If $f \in C^{2 m+1}[c, d]$ and $x \in[c, d]$, then for the remainder (18) we get

$$
\begin{aligned}
& \left|R_{a, m}[f ; a, b](x)\right| \\
& \leqslant \begin{cases}C^{\prime}(m)\left\|f^{(2 m+1)}\right\|_{\infty}(b-x)^{2 m+1}, & c<x<a, \\
C^{\prime}(m)\left\|f^{(2 m+1)}\right\|_{\infty}(b-a)^{2 m+1}, & a<x<b, \\
C^{\prime}(m)\left\|f^{(2 m+1)}\right\|_{\infty}(x-a)^{2 m+1}, & b<x<d,\end{cases}
\end{aligned}
$$

where $\|\cdot\|_{\infty}$ denotes the sup-norm on $[c, d]$ and

$$
\begin{array}{r}
C^{\prime}(m)=\frac{1}{(2 m+1) !}\left[2^{2 m}+\sum_{j=1}^{m} \sum_{k=1}^{2 j-1} \frac{2^{k+1}-2}{2 m-2 j+2}\left(\begin{array}{c}
2 m+1 \\
2 j
\end{array}\right)\right. \\
\left.\times\left(\begin{array}{c}
2 j \\
k
\end{array}\right)\left|B_{k}(1)\right|\right] .
\end{array}
$$

\section{The Modified Even Order Bernoulli-Type Quasi-Interpolants}

The multiquadric quasi-interpolant $\mathscr{L}_{B}[6]$ is defined by the following:

$$
\begin{aligned}
\mathscr{L}_{B}[f ; a, b](x)= & f\left(x_{0}\right) \psi_{0}(x)+\sum_{i=1}^{n-1} f\left(x_{i}\right) \psi_{i}(x) \\
& +f\left(x_{n}\right) \psi_{n}(x), \quad x \in[a, b],
\end{aligned}
$$


where

$$
\begin{aligned}
& \psi_{0}(x)=\frac{1}{2} c^{2} \int_{-\infty}^{x_{0}} \frac{1}{\left[(x-t)^{2}+c^{2}\right]^{3 / 2}} d t \\
& +\frac{1}{2} c^{2} \int_{x_{0}}^{x_{1}} \frac{\left(x_{1}-t\right) /\left(x_{1}-x_{0}\right)}{\left[(x-t)^{2}+c^{2}\right]^{3 / 2}} d t \\
& =\frac{1}{2}+\frac{\phi_{1}(x)-\phi_{0}(x)}{2\left(x_{1}-x_{0}\right)}, \\
& \psi_{n}(x)=\frac{1}{2} c^{2} \int_{x_{n}}^{\infty} \frac{1}{\left[(x-t)^{2}+c^{2}\right]^{3 / 2}} \\
& +\frac{1}{2} c^{2} \int_{x_{n-1}}^{x_{n}} \frac{\left(t-x_{n-1}\right) /\left(x_{n}-x_{n-1}\right)}{\left[(x-t)^{2}+c^{2}\right]^{3 / 2}} d t \\
& =\frac{1}{2}-\frac{\phi_{n}(x)-\phi_{n-1}(x)}{2\left(x_{n}-x_{n-1}\right)} \text {, } \\
& \psi_{i}(x)=\frac{1}{2} c^{2} \int_{x_{i-1}}^{x_{i+1}} \frac{B_{i}(t)}{\left[(x-t)^{2}+c^{2}\right]^{3 / 2}} d t \\
& =\frac{\phi_{i+1}(x)-\phi_{i}(x)}{2\left(x_{i+1}-x_{i}\right)}-\frac{\phi_{i}(x)-\phi_{i-1}(x)}{2\left(x_{i}-x_{i-1}\right)}
\end{aligned}
$$

for $i=1, \ldots, n-1$, where $\left\{B_{i}(t): t \in \mathbb{R}\right\}$ is the hat function that has the nodes $\left\{x_{i-1}, x_{i}, x_{i+1}\right\}$, that is identically zero outside the interval $x_{i-1} \leqslant t \leqslant x_{i+1}$ and that satisfies the normalization condition $B_{i}\left(x_{i}\right)=1$. The operator $\mathscr{L}_{B}$ reproduces constants. Based on the operator $\mathscr{L}_{B}$, we first define a family of even order Bernoulli-type multiquadric quasi-interpolants $\widetilde{\mathscr{L}}_{v_{m}}$ as follows:

$$
\begin{array}{r}
\widetilde{\mathscr{L}}_{v_{m}}[f ; a, b](x)=\sum_{i=0}^{n} \psi_{i}(x) P_{x_{i}, m}\left[f ; x_{i}, x_{i+1}\right](x), \\
x \in[a, b],
\end{array}
$$

where $P_{x_{i}, m}\left[f ; x_{i}, x_{i+1}\right](x)$ is the natural extension of the polynomial expansion defined in (14) and $x_{n+1}=x_{n-1}$. The operators $\widetilde{\mathscr{L}}_{v_{m}}$ possess the polynomial reproduction property as follows.

Theorem 4. The operators $\widetilde{\mathscr{L}}_{v_{m}}$ reproduce all univariate polynomials of degree no more than $2 \mathrm{~m}$.

Proof. The argument $\widetilde{\mathscr{L}}_{v_{m}}[p ; a, b](x)=p$ follows from the well-known property

$$
\sum_{i=0}^{n} \psi_{i}(x)=1
$$

since $P_{x_{i}, m}\left[p ; x_{i}, x_{i+1}\right](x)=p$ for $i=0, \ldots, n$, where $p \in \mathbb{P}^{2 m}$.

Although the quasi-interpolants $\widetilde{\mathscr{L}}_{v_{m}}$ reproduce all polynomials of degree $\leqslant 2 m$, they require the derivative of $f$ at every node, which are very difficult to measure in practice. Therefore, we use divided difference operator $D_{A}^{2 j-1} f$ in following Definition 5 to approximate $f^{(2 j-1)}$ in the operators $\widetilde{\mathscr{L}}_{v_{m}}$ and then get a family of modified even order Bernoullitype multiquadric quasi-interpolants $\mathscr{L}_{v_{m}}$.

Definition 5 (see [18]). Let $\mathscr{F}=\{f \mid f: \mathbb{R} \rightarrow \mathbb{R}\}$ and let $A$ be a discrete subset of $\mathbb{R}, j \in \mathbb{N}$. Suppose that $D^{2 j-1}$ is the order $2 j-1$ derivative. An operator $D_{A}^{2 j-1}: \mathscr{F} \rightarrow \mathscr{F}$ is said to be a $\mathbb{P}_{2 m}$-exact $A$-discretization of $D^{2 j-1}$ if and only if

(i) there exists a real vector $\lambda=\left(\lambda_{a}\right)_{a \in A}$ s.t. for any $f \in$ $\mathscr{F}$,

$$
D_{A}^{2 j-1} f(\cdot)=\sum_{a \in A} \lambda_{a} f(\cdot+a), \quad j=1, \ldots, m
$$

(ii) for any $p \in \mathbb{P}_{2 m}$,

$$
D_{A}^{2 j-1} p=D^{2 j-1} p
$$

In such situation, we also say that $D_{A}^{2 j-1} f$ is a $\mathbb{P}_{2 m}$-exact $A$-discretization of $D^{2 j-1} f$. Let the points be distinct in the set $A$; then $D_{A}^{2 j-1}$ is determined uniquely.

Let $|\cdot|$ denote the number of elements in set. Let the points in set $A$ be distinct and $|A|=2 m+1$; then by Definition 5 and [18], a $\mathbb{P}_{2 m}$-exact $A$-discretization of the order $2 j-1$ derivative $f^{(2 j-1)}$ is

$$
D_{A}^{2 j-1} f(x)=\sum_{a \in A} \lambda_{a} f(x+a), \quad j=1, \ldots, m,
$$

where

$$
\lambda_{a}=\frac{(-1)^{2 m+1-2 j}(2 j-1) ! \sum_{A^{\prime} \subset A \backslash\{a\},\left|A^{\prime}\right|=2 m+1-2 j} \prod_{c \in A^{\prime}}(c)}{\prod_{c \in A \backslash\{a\}}(a-c)} .
$$

By virtue of the location of each pair $x_{i}, x_{i+1}(i=0, \ldots, n)$, we choose suitable sets $A_{x_{i}}, A_{x_{i+1}}$ and then replace $f^{(2 j-1)}\left(x_{i}\right)$ and $f^{(2 j-1)}\left(x_{i+1}\right)$ with $D_{A_{x_{i}}}^{2 j-1} f\left(x_{i}\right)$ and $D_{A_{x_{i+1}}}^{2 j-1} f\left(x_{i+1}\right)$, respectively. Thus, the modification quasi-interpolants $\mathscr{L}_{v_{m}}$ can be expressed as follows:

$$
\begin{aligned}
& \mathscr{L}_{v_{m}}[f ; a, b](x) \\
& =\sum_{i=0}^{n} \psi_{i}(x)
\end{aligned}
$$

$$
\times\left[f\left(x_{i}\right)+\sum_{j=1}^{m}\left[\left(x_{i+1}-x_{i}\right)^{2 j-1} D_{A_{x_{i+1}}^{2 j-1}} f\left(x_{i+1}\right) \frac{2^{2 j}}{(2 j) !}\right.\right.
$$




$$
\begin{gathered}
\times \sum_{k=0}^{2 j-1}\left(\begin{array}{c}
2 j \\
k
\end{array}\right) B_{k}\left(\frac{1}{2}\right)\left(\frac{x-x_{i}}{2\left(x_{i+1}-x_{i}\right)}\right)^{2 j-k} \\
-\left(x_{i+1}-x_{i}\right)^{2 j-1} D_{A_{x_{i}}}^{2 j-1} f\left(x_{i}\right) \frac{2^{2 j}}{(2 j) !} \\
\left.\left.\times \sum_{k=0}^{2 j-1}\left(\begin{array}{c}
2 j \\
k
\end{array}\right) B_{k}(1)\left(\frac{x_{i}-x}{2\left(x_{i+1}-x_{i}\right)}\right)^{2 j-k}\right]\right], \\
x_{n+1}=x_{n-1} .
\end{gathered}
$$

Note that the expressions of $D_{A_{x_{i}}}^{2 j-1} f\left(x_{i}\right)(i=0, \ldots, n, j=$ $1, \ldots, m)$ in the modification operators $\mathscr{L}_{v_{m}}$ are provided by the following theorem.

Theorem 6. For any $A \subset \mathbb{R}$ and $x \in \mathbb{R}$, let $A$ be a $\mathbb{P}_{2 m^{-}}$ unisolvent set and $A_{x}=A-x$ denote the set of points $e \in \mathbb{R}$ of the form $e=a-x$, where $a \in A$. Let $A=\left\{x_{i}, \ldots, x_{i+2 m}\right\}(i=$ $0, \ldots, m-1),\left\{x_{i-m}, \ldots, x_{i}, \ldots, x_{i+m}\right\}(i=m, \ldots, n-m)$, and $\left\{x_{i-2 m}, \ldots, x_{i}\right\}(i=n-m+1, \ldots, n)$. Then, for each $j=1, \ldots, m$, we have

$$
D_{A_{x_{i}}}^{2 j-1} f\left(x_{i}\right)=\sum_{a \in A} \lambda_{a-x_{i}} f(a), \quad i=0, \ldots, n,
$$

where

$$
\begin{aligned}
& \lambda_{a-x_{i}} \\
& =\frac{(-1)^{2 m+1-2 j}(2 j-1) ! \sum_{A^{\prime} \subset A \backslash\{a\},\left|A^{\prime}\right|=2 m+1-2 j} \prod_{c \in A^{\prime}}\left(c-x_{i}\right)}{\prod_{c \in A \backslash\{a\}}(a-c)} .
\end{aligned}
$$

Proof. For each $x \in \mathbb{R}$, we set $A_{x}=\left\{x_{i}-x, \ldots, x_{i+2 m}-x\right\}(i=$ $0, \ldots, m-1),\left\{x_{i-m}-x, \ldots, x_{i}-x, \ldots, x_{i+m}-x\right\}(i=m, \cdots, n-$ $m)$, and $\left\{x_{i-2 m}-x, \ldots, x_{i}-x\right\}(i=n-m+1, \ldots, n)$. According to (50) and (53), we get

$$
D_{A_{x}}^{2 j-1} f(0)=\sum_{a \in A} \lambda_{a-x} f(a-x)
$$

where

$$
\begin{aligned}
& \lambda_{a-x} \\
& =\frac{(-1)^{2 m+1-2 j}(2 j-1) ! \sum_{A^{\prime} \subset A \backslash\{a\},\left|A^{\prime}\right|=2 m+1-2 j} \prod_{c \in A^{\prime}}(c-x)}{\prod_{c \in A \backslash\{a\}}(a-c)} .
\end{aligned}
$$

Therefore, we have

$$
D_{A_{x}}^{2 j-1} f(x)=\sum_{a \in A} \lambda_{a-x} f(a) .
$$

Let us set $x=x_{i} ;$ then we get the proof of the Theorem 6 .
Remark 7. For $m=1$, we give the expression of the modification operator $\mathscr{L}_{v_{1}}$ as follows:

$$
\begin{aligned}
& \mathscr{L}_{v_{1}}[f ; a, b](x) \\
& =\left[f\left(x_{0}\right)-\left(\frac{1}{2} \frac{\left(x_{0}-x\right)^{2}}{x_{1}-x_{0}}+\left(x_{0}-x\right)\right) D_{3}^{1} f\left(x_{0}\right)\right] \psi_{0}(x) \\
& +\sum_{i=1}^{n-1}\left[f\left(x_{i}\right)-\left(\frac{1}{2} \frac{\left(x_{i}-x\right)^{2}}{x_{i+1}-x_{i}}+\left(x_{i}-x\right)\right) D_{3}^{1} f\left(x_{i}\right)\right] \psi_{i}(x) \\
& +\sum_{i=1}^{n-1} \frac{1}{2} \frac{\left(x-x_{i-1}\right)^{2}}{x_{i}-x_{i-1}} D_{3}^{1} f\left(x_{i}\right) \psi_{i-1}(x) \\
& +\frac{1}{2} \frac{\left(x-x_{n-1}\right)^{2}}{x_{n}-x_{n-1}} D_{3}^{1} f\left(x_{n}\right) \psi_{n-1}(x) \\
& +\left[f\left(x_{n}\right)-\left(\frac{1}{2} \frac{\left(x_{n}-x\right)^{2}}{x_{n-1}-x_{n}}+\left(x_{n}-x\right)\right) D_{3}^{1} f\left(x_{n}\right)\right] \psi_{n}(x) \\
& +\frac{1}{2} \frac{\left(x-x_{n}\right)^{2}}{x_{n-1}-x_{n}} D_{3}^{1} f\left(x_{n-1}\right) \psi_{n}(x),
\end{aligned}
$$

where

$$
\begin{aligned}
D_{3}^{1} f\left(x_{0}\right)= & -\frac{x_{1}+x_{2}-2 x_{0}}{\left(x_{0}-x_{1}\right)\left(x_{0}-x_{2}\right)} f\left(x_{0}\right) \\
& -\frac{x_{2}-x_{0}}{\left(x_{1}-x_{0}\right)\left(x_{1}-x_{2}\right)} f\left(x_{1}\right) \\
& -\frac{x_{1}-x_{0}}{\left(x_{2}-x_{0}\right)\left(x_{2}-x_{1}\right)} f\left(x_{2}\right), \\
D_{3}^{1} f\left(x_{i}\right)= & -\frac{x_{i+1}-x_{i}}{\left(x_{i-1}-x_{i}\right)\left(x_{i-1}-x_{i+1}\right)} f\left(x_{i-1}\right) \\
& -\frac{x_{i-1}+x_{i+1}-2 x_{i}}{\left(x_{i}-x_{i-1}\right)\left(x_{i}-x_{i+1}\right)} f\left(x_{i}\right) \\
& -\frac{x_{i-1}-x_{i}}{\left(x_{i+1}-x_{i-1}\right)\left(x_{i+1}-x_{i-1}\right)\left(x_{i+1}-x_{i}\right)} f\left(x_{i+1}\right), \\
& -\frac{x_{n-2}+x_{n-1}-2 x_{n}}{\left(x_{n}-x_{n-2}\right)\left(x_{n}-x_{n-1}\right)} f\left(x_{n}\right) . \\
D_{3}^{1} f\left(x_{n}\right)= & -\frac{x_{n-1}-x_{n}-x_{n}}{\left(x_{n-2}-x_{n-1}\right)\left(x_{n-2}-x_{n}\right)} f\left(x_{n-2}\right) \\
& -\frac{\left.x_{n-1}-x_{n-2}\right)\left(x_{n-1}-x_{n}\right)}{\left(x_{n-1},\right.} f\left(x_{n-1}\right) \\
& \\
& \\
& \\
& \\
&
\end{aligned}
$$




\subsection{The Polynomial Reproduction Properties of the Operators $\mathscr{L}_{v_{m}}$}

Theorem 8. The operators $\mathscr{L}_{v_{m}}$ reproduce all univariate polynomials of degree no more than $2 \mathrm{~m}$.

Proof. By using the proof of Theorem 4 and formulas (51)(54), we get the proof of Theorem 8 immediately.

3.2. The Convergence Rate of the Operators $\mathscr{L}_{v_{m}}$. In order to obtain the convergence rate of the modified multiquadric quasi-interpolants $\mathscr{L}_{v_{m}}$, we make use of the following notations:

$$
\begin{gathered}
I_{\rho}(x)=[x-\rho, x+\rho], \quad \rho>0, \\
h=\frac{1}{2} \inf \left\{\rho>0: \forall x \in[a, b], I_{\rho}(x) \cap X \neq \emptyset\right\}, \\
M=\max _{x \in[a, b]} \#\left(I_{h}(x) \cap X\right),
\end{gathered}
$$

where \#(.) denotes the cardinality function. So, $2 h=$ $\max _{1 \leqslant i \leqslant n}\left|x_{i}-x_{i-1}\right|$ and $M$ denotes the maximum number of points from $X$ contained in an interval $I_{h}(x)$. At first, for the quasi-interpolants $\widetilde{\mathscr{L}}_{v_{m}}$, we then give the error estimates as follows.

Theorem 9. Let c satisfy

$$
c \leqslant D r^{l},
$$

where $D$ is a positive constant and $l$ is a positive integer. Let $f(x) \in C^{2 m}[a, b] ;$ then

$$
\left\|\widetilde{\mathscr{L}}_{v_{m}}[f ; a, b](x)-f(x)\right\|_{\infty} \leqslant C^{\prime} M\left\|f^{(2 m)}(x)\right\|_{\infty} T_{l, m}(h),
$$

where

$$
T_{l, m}(h)= \begin{cases}h^{2 m}, & 2 m<2 l-1, \\ h^{2 l-1}, & 2 m \geqslant 2 l-1,\end{cases}
$$

and $C^{\prime}$ is a positive constant independent of $f, x$, and $X$.

Proof. Let each pair $x_{i}, x_{i+1} \in[a, b]$, be fixed and let $x_{i}<x_{i+1}$. For each $x \in[a, b]$ we make use of the following settings:

$$
\begin{gathered}
d\left[x_{i}, x_{i+1}\right](x)= \begin{cases}x_{i+1}-x, & x \leqslant x_{i}, \\
x_{i+1}-x_{i}, & x_{i} \leqslant x \leqslant x_{i+1}, \\
x-x_{i}, & x_{i+1} \leqslant x,\end{cases} \\
d^{2 m}\left[x_{i}, x_{i+1}\right](x)=\left(d\left[x_{i}, x_{i+1}\right](x)\right)^{2 m} .
\end{gathered}
$$

By applying (14) to (48), we obtain

$$
\begin{aligned}
\mid \widetilde{\mathscr{L}}_{v_{m}} & {[f ; a, b](x)-f(x) \mid } \\
& =\left|\sum_{i=0}^{n} \psi_{i}(x)\left(P_{x_{i}, m}\left[f ; x_{i}, x_{i+1}\right]\right)-f(x)\right|
\end{aligned}
$$

$$
\begin{aligned}
& \leqslant \sum_{i=0}^{n} \psi_{i}(x)\left|P_{x_{i}, m}\left[f ; x_{i}, x_{i+1}\right](x)-f(x)\right| \\
& \leqslant C(m)\left\|f^{(2 m)}(x)\right\|_{\infty} S_{l, m}(x),
\end{aligned}
$$

where

$$
S_{l, m}(x)=\sum_{i=0}^{n} \psi_{i}(x) d^{2 m}\left[x_{i}, x_{i+1}\right](x) .
$$

Assume that

$$
\begin{gathered}
N=\left[\frac{b-a}{2 h}\right]+1, \\
Q_{\rho}(u)=(u-\rho, u+\rho], \quad u \in[a, b], \rho>0, \\
T_{j}=Q_{h}(x-2 h j) \cup Q_{h}(x+2 h j), \quad j=0, \ldots, N,
\end{gathered}
$$

where the set $\bigcup_{j=-N}^{N} Q_{h}(x+2 h j)$ denotes the covering of $[a, b]$ with half open intervals. Thus, for every $i \in\{0, \ldots, n\}$, there exists a unique $j \in\{0, \ldots, N\}$ s.t. $x_{i} \in T_{j}$. Then, we get the following inequalities:

$$
\begin{gathered}
(2 j-1) h \leqslant\left|x-x_{i}\right| \leqslant(2 j+1) h, \\
(2(j-1)-1) h \leqslant\left|x-\tau_{i}\right| \leqslant(2(j+1)+1) h,
\end{gathered}
$$

where $j=2, \ldots, N$ and $\tau_{i} \in\left[x_{i-1}, x_{i+1}\right]$. Therefore, we have from (70)

$$
d\left[x_{i}, x_{i+1}\right](x) \leqslant(2 j+3) h .
$$

We also obtain from the definition of $M$

$$
\begin{gathered}
1 \leqslant \#\left(X \cap T_{0}\right) \leqslant M, \\
1 \leqslant \#\left(X \cap T_{j}\right) \leqslant 2 M, \quad j=1, \ldots, N .
\end{gathered}
$$

On the other hand, when $x_{0} \in T_{j}, j=2, \ldots, N$, we get, after some calculations, by applying the first mean value theorem for integrals to (46),

$$
\begin{aligned}
\psi_{0}(x) \leqslant & \frac{1}{2} c^{2} \int_{-\infty}^{x_{0}} \frac{1}{|x-t|^{3}} d t+\frac{1}{2} c^{2} \frac{1}{\left[\left(x-\tau_{0}\right)^{2}+c^{2}\right]^{3 / 2}} \\
& \times \int_{x_{0}}^{x_{1}} \frac{x_{1}-t}{x_{1}-x_{0}} d t \\
\leqslant & \frac{1}{4} c^{2}\left|x-x_{0}\right|^{-2}+\frac{1}{4} c^{2}\left(x_{1}-x_{0}\right)\left|x-\tau_{0}\right|^{-3} \\
\leqslant & \frac{1}{4} c^{2}\left[(2 j-1)^{-2} h^{-2}+2(2 j-3)^{-3} h^{-2}\right] \\
\leqslant & c^{2} h^{-2}(2 j-3)^{-2},
\end{aligned}
$$

where $\tau_{0} \in\left[x_{0}, x_{1}\right]$. When $x_{n} \in T_{j}, j=2, \ldots, N$, we obtain in an analogous manner

$$
\psi_{n}(x) \leqslant c^{2} h^{-2}(2 j-3)^{-2} .
$$


When $x_{i}(i=1, \ldots, n-1) \in y_{j}(j=2, \ldots, N)$, we also obtain

$$
\begin{aligned}
\psi_{i}(x) & \leqslant \frac{1}{2} c^{2} \frac{1}{\left[\left(x-\tau_{i}\right)^{2}+c^{2}\right]^{3 / 2}} \int_{x_{i-1}}^{x_{i+1}} B_{i}(t) d t \\
& \leqslant \frac{1}{4} c^{2}\left(x_{i+1}-x_{i-1}\right)\left|x-\tau_{i}\right|^{-3} \\
& \leqslant c^{2} h^{-2}(2 j-3)^{-2},
\end{aligned}
$$

where $\tau_{i} \in\left[x_{i-1}, x_{i+1}\right]$. Then, for (68), we have

$$
\begin{aligned}
S_{l, m}(x)= & \sum_{x_{i} \in T_{0}, T_{1}} \psi_{i}(x) d^{2 m}\left[x_{i}, x_{i+1}\right](x) \\
& +\sum_{j=2}^{N} \sum_{x_{i} \in T_{j}} \psi_{i}(x) d^{2 m}\left[x_{i}, x_{i+1}\right](x) \\
\leqslant & \sum_{x_{i} \in T_{0}, T_{1}} d^{2 m}\left[x_{i}, x_{i+1}\right](x) \\
& +\sum_{j=2}^{N} \sum_{x_{i} \in T_{j}} \psi_{i}(x) d^{2 m}\left[x_{i}, x_{i+1}\right](x) \\
\leqslant & M(3 h)^{2 m}+2 M(5 h)^{2 m} \\
& +2 M \sum_{j=2}^{N} c^{2} h^{-2}(2 j-3)^{-2}((2 j+3) h)^{2 m} \\
\leqslant & 2 M(5 h)^{2 m}+2 M(5 h)^{2 m}+2 M \sum_{j=1}^{N} c^{2} h^{-2} j^{-2}((5 j) h)^{2 m} \\
\leqslant & 2 M 5^{2 m}\left(2 h^{2 m}+D^{2} h^{2 m-2+2 l} \sum_{j=1}^{N} j^{2 m-2}\right)
\end{aligned}
$$

where the last inequality follows from

$$
\begin{gathered}
2 j-3 \geqslant j, \quad j=3,4, \ldots, \\
2 j+3 \leqslant 5 j, \quad j=1,2, \ldots, \\
7^{2 m} \leqslant 5^{2 m}+\frac{1}{2^{2}} 10^{2 m}, \quad m=1,2, \ldots .
\end{gathered}
$$

Let $2 m<2 l-1$; then $2 h^{2 m}+D^{2} h^{2 l+2 m-2} \sum_{j=1}^{N} j^{2 m-2}=\mathcal{O}\left(h^{2 m}\right)$.

Let $2 m \geqslant 2 l-1$; then $2 h^{2 m}+D^{2} h^{2 l+2 m-2} \sum_{j=1}^{N} j^{2 m-2}=$ $\mathcal{O}\left(h^{2 l-1}\right)$.

Applying Theorem 3, we can obtain the desired error estimates of the operator $\widetilde{\mathscr{L}}_{v_{m}}$ in an analogous manner.

Theorem 10. Let c satisfy

$$
c \leqslant D h^{l}
$$

where $D$ is a positive constant and $l$ is a positive integer. Let $f(x) \in C^{2 m+1}[a, b]$; then

$$
\left\|\widetilde{\mathscr{L}}_{v_{m}}[f ; a, b](x)-f(x)\right\|_{\infty} \leqslant C^{\prime \prime} M\left\|f^{(2 m+1)}(x)\right\|_{\infty} T_{l, m}^{\prime}(h),
$$

where

$$
T_{l, m}^{\prime}(h)= \begin{cases}h^{2 m+1}, & 2 m+1<2 l-1, \\ h^{2 l-1}, & 2 m+1 \geqslant 2 l-1,\end{cases}
$$

and $C^{\prime \prime}$ is a positive constant independent of $f, x$, and $X$.

Because of disadvantage with the derivatives in the operators $\widetilde{\mathscr{L}}_{v_{m}}$, we give the following desired error estimates of the modification quasi-interpolants $\mathscr{L}_{v_{m}}$.

Theorem 11. Let c satisfy

$$
c \leqslant D h^{l},
$$

where $D$ is a positive constant and $l$ is a positive integer. Let $f(x) \in C^{2 m+1}[a, b]$; then

$$
\left\|\mathscr{L}_{v_{m}}[f ; a, b](x)-f(x)\right\|_{\infty} \leqslant C M\left\|f^{(2 m+1)}(x)\right\|_{\infty} T_{l, m}^{\prime}(h),
$$

where

$$
T_{l, m}^{\prime}(h)= \begin{cases}h^{2 m+1}, & 2 m+1<2 l-1, \\ h^{2 l-1}, & 2 m+1 \geqslant 2 l-1,\end{cases}
$$

and $C$ is a positive constant independent of $f, x$, and $X$.

Proof. Consider

$$
\begin{aligned}
\left|\mathscr{L}_{v_{m}}[f ; a, b](x)-f(x)\right| & \\
\leqslant & \left|\mathscr{L}_{v_{m}}[f ; a, b](x)-\widetilde{\mathscr{L}}_{v_{m}}[f ; a, b](x)\right| \\
& +\left|\widetilde{\mathscr{L}}_{v_{m}}[f ; a, b](x)-f(x)\right| .
\end{aligned}
$$

The second term of the right-hand sides in (84) has been obtained from Theorem 10, so we only need to prove the first term.

We denote by $h_{\max }$ and $h_{\min }$ the maximum and the minimum distance between adjacent nodes, respectively. Let $h=h_{\max } / h_{\min } \geqslant 1$ and $C_{0}=\left\|f^{(2 m+1)}(x)\right\|_{\infty}$.

Let $C_{1}$ be a constant; then, according to [18], we get

$$
\begin{aligned}
\sum_{a \in A}\left|\lambda_{a}\right| \frac{|a|^{2 m+1}}{(2 m+1) !} & \leqslant \sum_{a \in A}\left|\lambda_{a}\right| \frac{1}{(2 m+1) !}\left(\max _{a \in A}|A|\right)^{2 m+1} \\
& \leqslant C_{1} h^{2 m+1-(2 j-1)} k^{2 m} .
\end{aligned}
$$

Therefore, we obtain

$$
\begin{aligned}
\left|D_{A}^{2 j-1} f\left(x_{i}\right)-f^{(2 j-1)}\left(x_{i}\right)\right| \leqslant & C_{1} h^{2 m+1-(2 j-1)} k^{2 m} \\
& \times\left\|f^{(2 m+1)}(x)\right\|_{\infty} .
\end{aligned}
$$


TABLE 1: Numerical results of the operators $S_{B_{m}}$ and $\mathscr{L}_{v_{m}}$ for the saddle function.

\begin{tabular}{cccccc}
\hline & $S_{B_{m}} f_{1}$ & & & $\mathscr{L}_{v_{m}} f_{1}$ \\
$(\mu, m)$ & $\varepsilon_{\text {mean }}$ & $\varepsilon_{\max }$ & $(l, m)$ & $\varepsilon_{\text {mean }}$ & $\varepsilon_{\text {max }}$ \\
\hline$(2,1)$ & $1.002 \times 10^{-3}$ & $4.786 \times 10^{-3}$ & $(2,1)$ & $2.237 \times 10^{-4}$ & $1.126 \times 10^{-3}$ \\
$(2,2)$ & $5.836 \times 10^{-4}$ & $3.654 \times 10^{-3}$ & $(2,2)$ & $3.356 \times 10^{-6}$ & $2.745 \times 10^{-5}$ \\
$(2,3)$ & $4.148 \times 10^{-4}$ & $2.776 \times 10^{-3}$ & $(2,3)$ & $4.020 \times 10^{-7}$ & $2.300 \times 10^{-6}$ \\
$(3,1)$ & $4.100 \times 10^{-4}$ & $2.578 \times 10^{-3}$ & $(3,1)$ & $2.063 \times 10^{-4}$ & $1.097 \times 10^{-3}$ \\
$(3,2)$ & $1.626 \times 10^{-4}$ & $1.200 \times 10^{-3}$ & $(3,2)$ & $1.698 \times 10^{-6}$ & $2.623 \times 10^{-5}$ \\
$(3,3)$ & $6.893 \times 10^{-5}$ & $2.332 \times 10^{-4}$ & $(3,3)$ & $1.325 \times 10^{-8}$ & $2.021 \times 10^{-7}$ \\
$(4,1)$ & $3.008 \times 10^{-4}$ & $3.643 \times 10^{-3}$ & $(4,1)$ & $1.824 \times 10^{-4}$ & $1.053 \times 10^{-3}$ \\
$(4,2)$ & $1.822 \times 10^{-4}$ & $2.004 \times 10^{-4}$ & $(4,2)$ & $9.620 \times 10^{-7}$ & $2.363 \times 10^{-5}$ \\
$(4,3)$ & $1.844 \times 10^{-5}$ & $5.491 \times 10^{-4}$ & $(4,3)$ & $9.891 \times 10^{-8}$ & $2.031 \times 10^{-7}$ \\
\hline
\end{tabular}

TABLE 2: Numerical results of the operators $\mathscr{L}_{H_{2 m-1}}$ and $\mathscr{L}_{v_{m}}$ for the saddle function.

\begin{tabular}{|c|c|c|c|c|c|}
\hline \multicolumn{3}{|c|}{$\mathscr{L}_{H_{2 m-1}} f_{1}$} & \multicolumn{3}{|c|}{$\mathscr{L}_{v_{m}} f_{1}$} \\
\hline$(l, m)$ & $\varepsilon_{\text {mean }}$ & $\varepsilon_{\max }$ & $(l, m)$ & $\varepsilon_{\text {mean }}$ & $\varepsilon_{\max }$ \\
\hline$(2,1)$ & $2.654 \times 10^{-4}$ & $1.200 \times 10^{-3}$ & $(2,1)$ & $2.237 \times 10^{-4}$ & $1.126 \times 10^{-3}$ \\
\hline$(2,2)$ & $6.678 \times 10^{-6}$ & $4.005 \times 10^{-5}$ & $(2,2)$ & $3.356 \times 10^{-6}$ & $2.745 \times 10^{-5}$ \\
\hline$(2,3)$ & $3.400 \times 10^{-6}$ & $1.280 \times 10^{-5}$ & $(2,3)$ & $4.020 \times 10^{-7}$ & $2.300 \times 10^{-6}$ \\
\hline$(3,1)$ & $2.541 \times 10^{-4}$ & $1.180 \times 10^{-3}$ & $(3,1)$ & $2.063 \times 10^{-4}$ & $1.097 \times 10^{-3}$ \\
\hline$(3,2)$ & $6.435 \times 10^{-6}$ & $4.160 \times 10^{-5}$ & $(3,2)$ & $1.698 \times 10^{-6}$ & $2.623 \times 10^{-5}$ \\
\hline$(3,3)$ & $1.236 \times 10^{-7}$ & $1.230 \times 10^{-6}$ & $(3,3)$ & $1.325 \times 10^{-8}$ & $2.021 \times 10^{-7}$ \\
\hline$(4,1)$ & $2.540 \times 10^{-4}$ & $1.180 \times 10^{-3}$ & $(4,1)$ & $1.824 \times 10^{-4}$ & $1.053 \times 10^{-3}$ \\
\hline$(4,2)$ & $6.435 \times 10^{-6}$ & $4.160 \times 10^{-5}$ & $(4,2)$ & $9.620 \times 10^{-7}$ & $2.363 \times 10^{-5}$ \\
\hline$(4,3)$ & $1.185 \times 10^{-7}$ & $1.230 \times 10^{-6}$ & $(4,3)$ & $9.891 \times 10^{-8}$ & $2.031 \times 10^{-7}$ \\
\hline
\end{tabular}

Let $C_{2}, C_{3}, \ldots, C_{2 m}, \bar{C}, \underline{C}, C$ be constants; then

$$
\begin{aligned}
& \mid \mathscr{L}_{v_{m}}[f ; a, b](x)-\widetilde{\mathscr{L}}_{v_{m}}[f ; a, b](x) \mid \\
& \leqslant C_{0} C_{2} h^{2 m} k^{2 m} \sum_{i=0}^{n}\left|x-x_{i}\right| \psi_{i}(x)+C_{0} C_{3} h^{2 m-1} k^{2 m} \\
& \quad \times \sum_{i=0}^{n}\left|x-x_{i}\right|^{2} \psi_{i}(x)+\cdots+C_{0} C_{2 m} h^{2} k^{2 m} \\
& \times \sum_{i=0}^{n}\left|x-x_{i}\right|^{2 m-1} \psi_{i}(x)
\end{aligned}
$$$$
\leqslant C_{0} C_{2} h^{2 m} k^{2 m}
$$$$
\times\left(\sum_{x_{i} \in T_{0}, T_{1}}\left|x-x_{i}\right| \psi_{i}(x)+\sum_{j=2}^{N} \sum_{x_{i} \in T_{j}}\left|x-x_{i}\right| \psi_{i}(x)\right)
$$$$
+C_{0} C_{3} h^{2 m-1} k^{2 m}
$$$$
\times\left(\sum_{x_{i} \in T_{0}, T_{1}}\left|x-x_{i}\right|^{2} \psi_{i}(x)+\sum_{j=2}^{N} \sum_{x_{i} \in T_{j}}\left|x-x_{i}\right|^{2} \psi_{i}(x)\right)
$$

$$
\begin{aligned}
& +\cdots+C_{0} C_{2 m} h^{2} k^{2 m} \\
& \times\left(\sum_{x_{i} \in T_{0}, T_{1}}\left|x-x_{i}\right|^{2 m-1} \psi_{i}(x)+\sum_{j=2}^{N} \sum_{x_{i} \in T_{j}}\left|x-x_{i}\right|^{2 m-1} \psi_{i}(x)\right) \\
\leqslant & C_{0} C_{2} h^{2 m} k^{2 m} \\
\times & \left(M h+2 M(3 h)+2 M \sum_{j=2}^{N} c^{2} h^{-2}(2 j-3)^{-2}(2 j+1) h\right) \\
+ & \left.C_{0} C_{3} h^{2 m-1} k^{2 m}\right) \\
\times & \left(M h^{2}+2 M(3 h)^{2}+\sum_{j=2}^{N} c^{2} h^{-2}(2 j-3)^{-2}((2 j+1) h)^{2}\right) \\
+ & \ldots+C_{0} C_{2 m} h^{2} k^{2 m} \\
\times & \left(M h^{2 m-1}+2 M(3 h)^{2 m-1}\right. \\
\leqslant & =M\left\|f^{(2 m+1)}(x)\right\|_{\infty}\left(h^{2 m+1}+c^{2} h^{2 m-2}+c^{2} h^{2 m-3}+\cdots+c^{2} h\right) \\
& \left.+2 M \sum_{j=2}^{N} c^{2} h^{-2}(2 j-3)^{-2}((2 j+1) h)^{2 m-1}\right)
\end{aligned}
$$


TABLE 3: Numerical results of the operators $S_{B_{m}}$ and $\mathscr{L}_{v_{m}}$ for the sphere function.

\begin{tabular}{cccccc}
\hline & $S_{B_{m}} f_{2}$ & & & $\mathscr{L}_{v_{m}} f_{2}$ \\
$(\mu, m)$ & $\varepsilon_{\text {mean }}$ & $\varepsilon_{\max }$ & $(l, m)$ & $(2,1)$ & $\varepsilon_{\text {mean }}$ \\
\hline$(2,1)$ & $2.012 \times 10^{-3}$ & $6.732 \times 10^{-3}$ & $(2,2)$ & $2.356 \times 10^{-4}$ & $4.664 \times 10^{-4}$ \\
$(2,2)$ & $1.521 \times 10^{-4}$ & $9.474 \times 10^{-4}$ & $(2,3)$ & $901 \times 10^{-6}$ & $2.045 \times 10^{-6}$ \\
$(2,3)$ & $1.900 \times 10^{-4}$ & $8.204 \times 10^{-4}$ & $(3,1)$ & $2.097 \times 10^{-8}$ & $9.763 \times 10^{-7}$ \\
$(3,1)$ & $4.834 \times 10^{-4}$ & $1.255 \times 10^{-3}$ & $(3,2)$ & $9.972 \times 10^{-4}$ & $4.024 \times 10^{-4}$ \\
$(3,2)$ & $2.801 \times 10^{-5}$ & $1.189 \times 10^{-4}$ & $(3,3)$ & $8.851 \times 10^{-9}$ & $3.665 \times 10^{-7}$ \\
$(3,3)$ & $2.711 \times 10^{-5}$ & $1.080 \times 10^{-4}$ & $(4,1)$ & $2.636 \times 10^{-4}$ & $3.541 \times 10^{-9}$ \\
$(4,1)$ & $3.991 \times 10^{-4}$ & $1.332 \times 10^{-3}$ & $(4,2)$ & $8.614 \times 10^{-8}$ & $3.964 \times 10^{-4}$ \\
$(4,2)$ & $1.989 \times 10^{-5}$ & $1.278 \times 10^{-4}$ & $(4,3)$ & $9.811 \times 10^{-9}$ & $3.524 \times 10^{-7}$ \\
$(4,3)$ & $1.249 \times 10^{-5}$ & $5.604 \times 10^{-5}$ & & $2.004 \times 10^{-8}$ \\
\hline
\end{tabular}

$$
\begin{aligned}
& \leqslant \underline{C} M\left\|f^{(2 m+1)}(x)\right\|_{\infty}\left(h^{2 m+1}+c^{2} h\right) \\
& \leqslant \underline{C} M\left\|f^{(2 m+1)}(x)\right\|_{\infty}\left(h^{2 m+1}+D^{2} h^{2 l+1}\right) .
\end{aligned}
$$

Finally, applying Theorem 10 and (87) to (84), we get

$$
\begin{aligned}
\left|\mathscr{L}_{v_{m}}[f ; a, b](x)-f(x)\right| \leqslant & C M\left\|f^{(2 m+1)}(x)\right\|_{\infty} \\
& \times\left(2 h^{2 m+1}+D^{2} h^{2 l+1}+D^{2} h^{2 l-1}\right) .
\end{aligned}
$$

Let $2 m+1<2 l-1$, then $2 h^{2 m+1}+D^{2} h^{2 l-1}=\mathcal{O}\left(h^{2 m+1}\right)$. Let $2 m+1 \geqslant 2 l-1$, then $2 h^{2 m+1}+D^{2} h^{2 l-1}=\mathcal{O}\left(h^{2 l-1}\right)$.

\section{Numerical Examples}

We consider the following functions on the interval $[0,1]$, which are firstly used in [16]:

$$
\begin{gathered}
\text { Saddle } f_{1}=\frac{1.25}{6+6(3 x-1)^{2}}, \\
\text { Sphere } f_{2}=\frac{\sqrt{64-81(x-0.5)^{2}}}{9}-0.5 \text {. }
\end{gathered}
$$

We apply the interpolation operators $S_{B_{m}}$, the quasiinterpolation operators $\mathscr{L}_{H_{2 m-1}}$, and the quasi-interpolants $\mathscr{L}_{v_{m}}$ on the above functions with $c=(2 h)^{l}$, where $S_{B_{m}}$ and $\mathscr{L}_{H_{2 m-1}}$ are defined by $[10,16]$, respectively.

We use uniform grids of 21 points for the operators $S_{B_{m}}$, $\mathscr{L}_{H_{2 m-1}}$, and $\mathscr{L}_{v_{m}}$ in Tables $1,2,3$, and 4 . In order to estimate the errors as accurate as possible, we compute the approximation functions at the points $i / 101, i=1, \ldots, 100$. Tables 1-4 show the mean and max errors which are computed for different values of the parameters $\mu, l$, and $m$. The numerical results show that our quasi-interpolants $\mathscr{L}_{v_{m}}$ have better approximation behavior.

\section{An Application of the New Operators}

After solving the following initial value problems:

$$
\begin{gathered}
y^{\prime}(x)=f(x, y(x)), \quad(x, y) \in[a, b] \times R, \\
y\left(x_{0}\right)=y_{0}, \quad x_{0} \in[a, b],
\end{gathered}
$$

by virtue of a discrete method, we often need to master the solution on a set of points that differs from the grid. Here we use our operators $\mathscr{L}_{v_{m}}$ to solve the problems. In fact, combinations of our operators $\mathscr{L}_{v_{m}}$ with discrete solvers of ODEs provide approximations of the solution of the problems (90) on $[a, b]$. An algorithm for constructing these quasiinterpolants is given as follows. The discrete solver produces an approximation $\tilde{y}_{i}$ of the exact solution $y\left(x_{i}\right)$ at nodes $x_{i}, i=0, \ldots, n$ in $[a, b]$. Substituting the exact values mentioned above into the definition of our operators $\mathscr{L}_{v_{m}}$ by their respective approximations, we get the proposed quasi-interpolants. We consider the initial value problems as follows.

\section{Problem A}

$$
\begin{gathered}
y^{\prime}(x)=x e^{-2 x}-2 y, \quad(x, y) \in[0,1] \times \mathbb{R}, \\
y(0)=-1 .
\end{gathered}
$$

\section{Problem B}

$$
\begin{gathered}
y^{\prime}(x)=\sin (2 x)-y \tan (x), \quad(x, y) \in[0,1] \times \mathbb{R}, \\
y(0)=-2 .
\end{gathered}
$$

The exact solutions of Problems A and B are $y(x)=(x-$ 1) $e^{x}$ and $y(x)=-2 \cos ^{2} x$, respectively. By the Runge-Kutta method of order 4 , we obtain the $\tilde{y}_{i}$ on a uniform grid of 21 nodes in $[0,1]$. Calculating the approximative functions at points $i / 101, i=1, \ldots, 100$, we get the mean and $\max$ errors in Table 5. Comparing the approximation capacity of our proposed quasi-interpolants with that of Runge-Kutta scheme of order 4 and Wang et al.s quasi-interpolation scheme [10] in Table 5, we find that our technique has smaller errors in the Problems A and B. 
TABLE 4: Numerical results of the operators $\mathscr{L}_{H_{2 m-1}}$ and $\mathscr{L}_{v_{m}}$ for the sphere function.

\begin{tabular}{cccccc}
\hline & $\mathscr{L}_{\mathrm{H}_{2 m-1}} f_{2}$ & & & $\mathscr{L}_{v_{m}} f_{2}$ & \\
$(l, m)$ & $\varepsilon_{\text {man }}$ & $\varepsilon_{\max }$ & $(l, m)$ & $\varepsilon_{\text {mean }}$ & $\varepsilon_{\max }$ \\
\hline$(2,1)$ & $3.037 \times 10^{-4}$ & $5.727 \times 10^{-4}$ & $(2,1)$ & $2.356 \times 10^{-4}$ & $4.664 \times 10^{-4}$ \\
$(2,2)$ & $1.590 \times 10^{-6}$ & $4.065 \times 10^{-6}$ & $(2,2)$ & $1.001 \times 10^{-6}$ & $2.045 \times 10^{-6}$ \\
$(2,3)$ & $9.838 \times 10^{-7}$ & $2.290 \times 10^{-6}$ & $(2,3)$ & $9.097 \times 10^{-8}$ & $9.763 \times 10^{-7}$ \\
$(3,1)$ & $2.848 \times 10^{-4}$ & $5.653 \times 10^{-4}$ & $(3,1)$ & $2.304 \times 10^{-4}$ & $4.024 \times 10^{-4}$ \\
$(3,2)$ & $2.217 \times 10^{-7}$ & $3.077 \times 10^{-6}$ & $(3,2)$ & $9.972 \times 10^{-8}$ & $3.665 \times 10^{-7}$ \\
$(3,3)$ & $1.581 \times 10^{-8}$ & $3.135 \times 10^{-8}$ & $(3,3)$ & $8.851 \times 10^{-9}$ & $3.541 \times 10^{-9}$ \\
$(4,1)$ & $2.848 \times 10^{-4}$ & $5.653 \times 10^{-4}$ & $(4,1)$ & $2.636 \times 10^{-4}$ & $3.964 \times 10^{-4}$ \\
$(4,2)$ & $6.017 \times 10^{-7}$ & $3.107 \times 10^{-6}$ & $(4,2)$ & $8.614 \times 10^{-8}$ & $3.524 \times 10^{-7}$ \\
$(4,3)$ & $1.730 \times 10^{-8}$ & $6.000 \times 10^{-8}$ & $(4,3)$ & $9.811 \times 10^{-9}$ & $2.004 \times 10^{-8}$ \\
\hline
\end{tabular}

TABle 5: Numerical results of the initial value Problems A and B.

\begin{tabular}{lcccc}
\hline & \multicolumn{2}{c}{ Problem A } & $\varepsilon_{\text {mean }}$ & Problem B \\
\hline$\tilde{y}_{i}$ & $\varepsilon_{\text {mean }}$ & $\varepsilon_{\text {max }}$ & $2.09 \times 10^{-6}$ & \\
$\mathscr{L}_{H_{2 m-1}},(l, m)=(4,2)$ & $6.34 \times 10^{-7}$ & $1.94 \times 10^{-6}$ & $7.00 \times 10^{-7}$ & $1.23 \times 10^{-5}$ \\
$\mathscr{L}_{H_{2 m-1}},(l, m)=(4,3)$ & $2.30 \times 10^{-7}$ & $6.18 \times 10^{-7}$ & $3.84 \times 10^{-7}$ & $2.43 \times 10^{-6}$ \\
$\mathscr{L}_{v_{m}},(l, m)=(4,2)$ & $8.05 \times 10^{-8}$ & $5.72 \times 10^{-7}$ & $9.32 \times 10^{-8}$ & $4.02 \times 10^{-6}$ \\
$\mathscr{L}_{v_{m}},(l, m)=(4,3)$ & $6.02 \times 10^{-8}$ & $9.17 \times 10^{-8}$ & $8.72 \times 10^{-8}$ & $3.43 \times 10^{-7}$ \\
\hline
\end{tabular}

\section{Conclusions}

In this paper, we propose a family of modified even order Bernoulli-type multiquadric quasi-interpolants $\mathscr{L}_{v_{m}}$ which reproduce polynomials of higher degree. There is no demand for the derivatives of function $f$ approximated at each node in our operators $\mathscr{L}_{v_{m}}$, so they do not increase the orders of smoothness of the function $f$. Under a certain assumption, we give an expected result on the convergence rate of our operators $\mathscr{L}_{v_{m}}$. The numerical examples show that our operators $\mathscr{L}_{v_{m}}$ produce higher degree of accuracy. Furthermore, applying the operators $\mathscr{L}_{v_{m}}$ to the fitting of discrete solutions of initial value problems, we find that our operators $\mathscr{L}_{v_{m}}$ provide more accurate approximation solver.

\section{Conflict of Interests}

The authors declare that there is no conflict of interests regarding the publication of this paper.

\section{Acknowledgment}

The work was supported by National Natural Science Foundation of China (Grant nos. 61373003 and 11271041).

\section{References}

[1] R. L. Hardy, "Multiquadric equations of topography and other irregular surfaces," Journal of Geophysical Research, vol. 76, no. 8, pp. 1905-1915, 1971.

[2] M. D. Buhmann, "Multivariate cardinal interpolation with radial-basis functions," Constructive Approximation, vol. 6, no. 3, pp. 225-255, 1990.
[3] M. D. Buhmann, "Multivariate interpolation in odddimensional Euclidean spaces using multiquadrics," Constructive Approximation, vol. 6, no. 1, pp. 21-34, 1990.

[4] R. Franke, "Scattered data interpolation: tests of some methods," Mathematics of Computation, vol. 38, no. 157, pp. 181-200, 1982.

[5] C. A. Micchelli, "Interpolation of scattered data: distance matrices and conditionally positive definite functions," Constructive Approximation, vol. 2, no. 1, pp. 11-22, 1986.

[6] R. K. Beatson and M. J. D. Powell, "Univariate multiquadric approximation: quasi-interpolation to scattered data," Constructive Approximation, vol. 8, no. 3, pp. 275-288, 1992.

[7] Z. M. Wu and R. Schaback, "Shape preserving properties and convergence of univariate multiquadric quasi-interpolation," Acta Mathematicae Applicatae Sinica, vol. 10, no. 4, pp. 441-446, 1994.

[8] L. Ling, "A univariate quasi-multiquadric interpolation with better smoothness," Computers \& Mathematics with Applications, vol. 48, no. 5-6, pp. 897-912, 2004.

[9] R. Feng and F. Li, "A shape-preserving quasi-interpolation operator satisfying quadratic polynomial reproduction property to scattered data," Journal of Computational and Applied Mathematics, vol. 225, no. 2, pp. 594-601, 2009.

[10] R.-H. Wang, M. Xu, and Q. Fang, "A kind of improved univariate multiquadric quasi-interpolation operators," Computers \& Mathematics with Applications, vol. 59, no. 1, pp. 451-456, 2010.

[11] R. Chen and Z. Wu, "Applying multiquadratic quasiinterpolation to solve Burgers' equation," Applied Mathematics and Computation, vol. 172, no. 1, pp. 472-484, 2006.

[12] R. Chen and Z. Wu, "Solving partial differential equation by using multiquadric quasi-interpolation," Applied Mathematics and Computation, vol. 186, no. 2, pp. 1502-1510, 2007.

[13] Y. C. Hon and Z. Wu, "A quasi-interpolation method for solving stiff ordinary differential equations," International Journal for 
Numerical Methods in Engineering, vol. 48, no. 8, pp. 1187-1197, 2000.

[14] Z. Wu, "Dynamically knots setting in meshless method for solving time dependent propagations equation," Computer Methods in Applied Mechanics and Engineering, vol. 193, no. 12-14, pp. 1221-1229, 2004.

[15] Z. Wu, "Dynamical knot and shape parameter setting for simulating shock wave by using multi-quadric quasi-interpolation," Engineering Analysis with Boundary Elements, vol. 29, no. 4, pp. 354-358, 2005.

[16] R. Caira and F. Dell'Accio, "Shepard-Bernoulli operators," Mathematics of Computation, vol. 76, no. 257, pp. 299-321, 2007.

[17] F. A. Costabile, F. Dell'Accio, and R. Luceri, "Explicit polynomial expansions of regular real functions by means of even order Bernoulli polynomials and boundary values," Journal of Computational and Applied Mathematics, vol. 176, no. 1, pp. 77-90, 2005.

[18] C. Rabut, "Multivariate divided differences with simple knots," SIAM Journal on Numerical Analysis, vol. 38, no. 4, pp. 12941311, 2001.

[19] R. Jordan, Calculus of Finite Differences, Chelsea, New York, NY, USA, 1960.

[20] P. J. Davis, Interpolation and Approximation, Dover, New York, NY, USA, 1975.

[21] http://functions.wolfram.com/05.14.16.0008.01. 


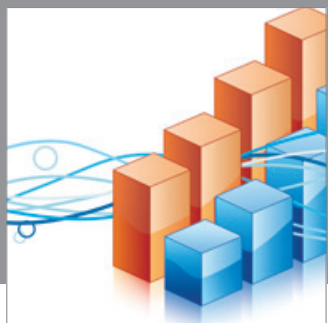

Advances in

Operations Research

mansans

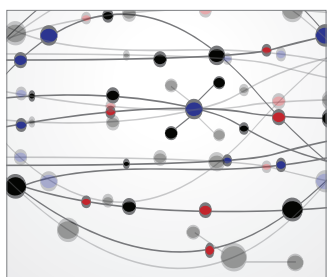

The Scientific World Journal
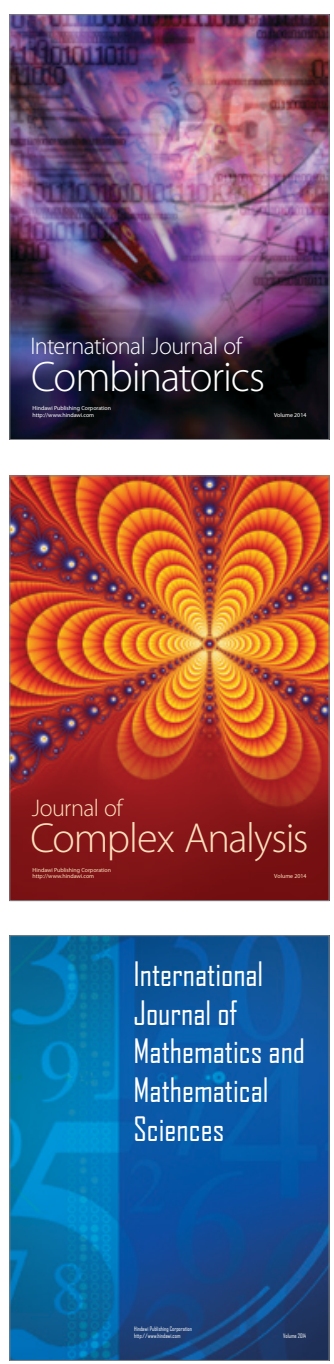
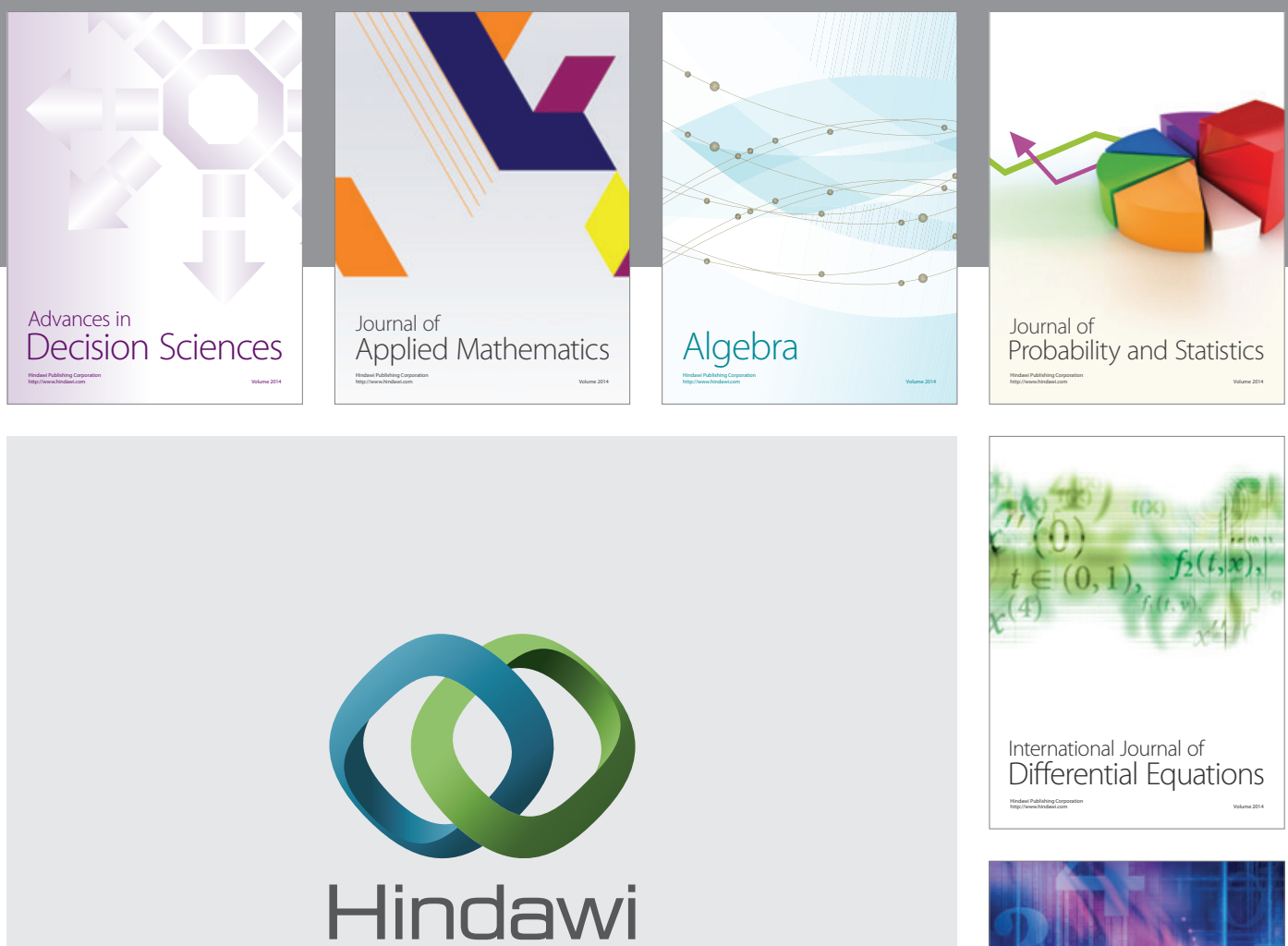

Submit your manuscripts at http://www.hindawi.com
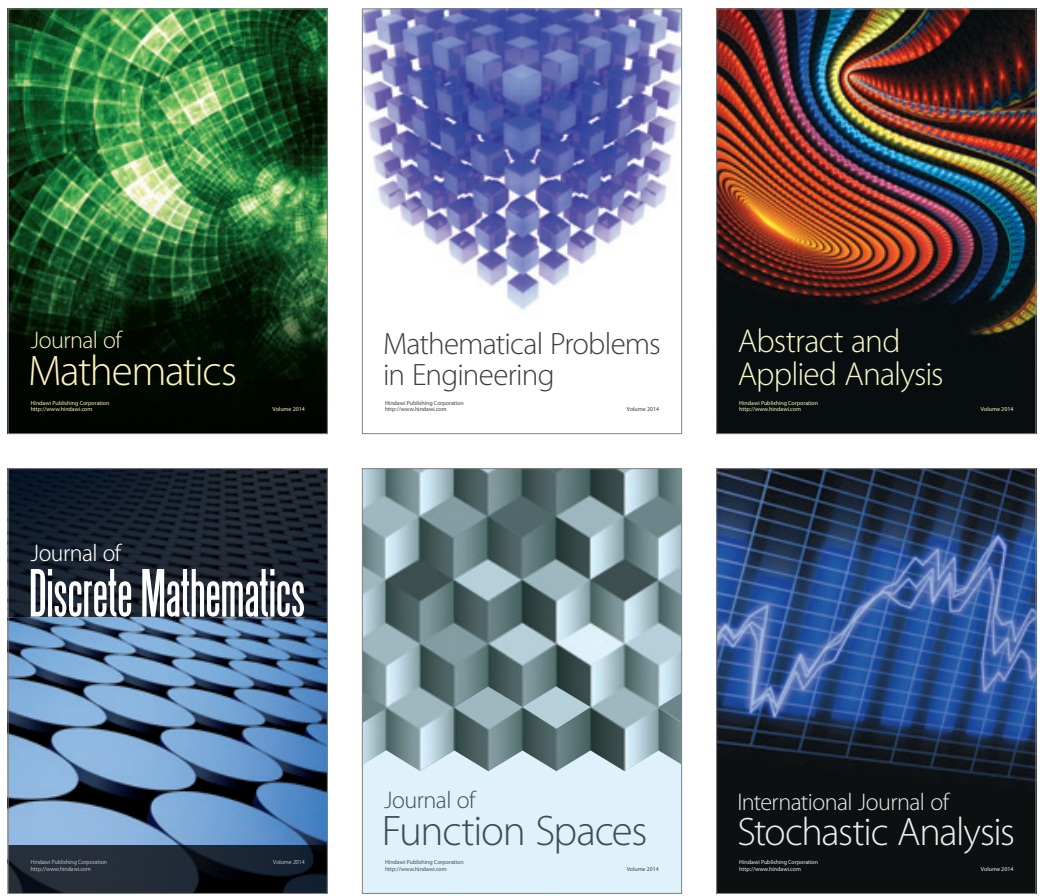

Journal of

Function Spaces

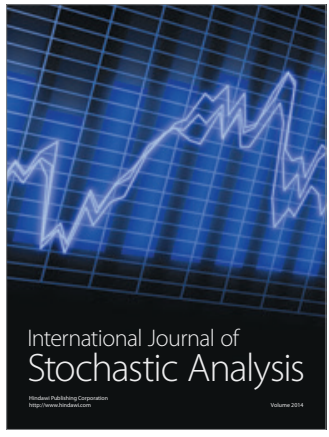

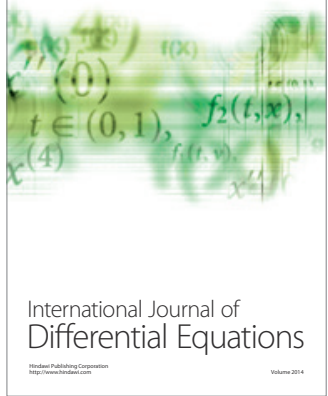
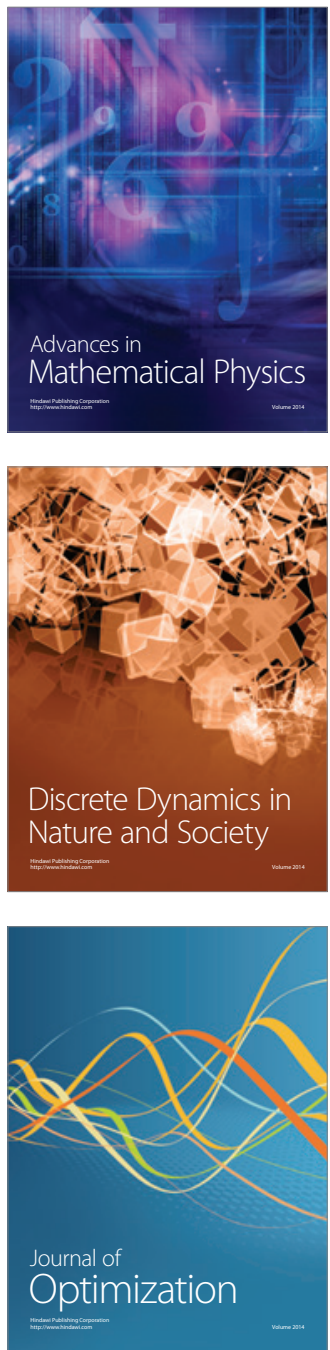